\title{
Verbal representation in task order control: An examination with transition and task cues in random task switching
}

\author{
ERINa Saeki and Satoru Saito \\ Kyoto University, Kyoto, Japan
}

\begin{abstract}
Recent task-switching studies in which a predictable task sequence has been used have indicated that verbal representation contributes to the control of task order information. The present study focused on the role of verbal representation in sequential task decisions, which are an important part of task order control, and examined the effects of articulatory suppression in a random-task-cuing paradigm with two different types of cues presented just before the presentation of a stimulus: a transition cue and a task cue. The former cue provided information only about switching or repeating the task, whereas the latter was associated directly with the identity of the task (i.e., indicating a parity or a magnitude task). In Experiment 1, in which transition cues guided task sequences, articulatory suppression impaired performance in both repetition and switch trials, thereby increasing the mixing costs. In Experiment 2, in which a task cue, rather than a transition cue, was presented to examine the influence of a cue-decoding process, articulatory suppression had no specific effect on task performance. Experiment 3 , in which the transition cue and the task cue were randomly presented in the same block to equalize the memory load and task strategy for the two types of cues, confirmed that articulatory suppression significantly increased the mixing costs only in transition cue trials. The results from the three experiments indicated that the use of verbal representation is effective in sequential task decision - that is, in selecting a task set on the basis of transient task order information in both repetition and switch trials.
\end{abstract}

Goal-directed behavior is founded on mechanisms that select and execute an appropriate task set, using relevant stimulus and response features conformable to that goal (Mayr, 2003). The nature and functions of task sets have been intensively examined with a task-switching paradigm (Monsell, 2003), in which participants are required to repeat the same task or to switch from one task to another. Generally, performance in task switching is worse (i.e., requires more time and is less accurate) than that in task repetition (e.g., Allport, Styles, \& Hsieh, 1994; Meiran, 1996; Rogers \& Monsell, 1995). A large body of literature exists on the task-switching paradigm. However, in this study, we focused on a specific issue suggested by previous studies - namely, that verbal representations are useful mediators for efficient task-switching performance, especially in predictable and sequential task switching (Baddeley, Chincotta, \& Adlam, 2001; Bryck \& Mayr, 2005; Emerson \& Miyake, 2003; Saeki \& Saito, 2004a, 2004b; Saeki, Saito, \& Kawaguchi, 2006).

One procedure that includes predictable and sequential task switching is the list paradigm, in which participants are required to repeat the same task or to switch between two tasks alternately in separate blocks (Jersild, 1927). The difference between reaction times (RTs) and errors in the task-switching block and those in the task repetition block is referred to as the alternation cost (Rubin \& Meiran, 2005). Recent studies in which the list paradigm has been used have examined the role of verbal representations in task switching through an articulatory suppression technique, which requires participants to continuously articulate task-irrelevant words that interfere with their use of speech-based verbal representation. The findings showed that articulatory suppression increased the alternation cost only when an external switching cue was not presented (Baddeley et al., 2001; Emerson \& Miyake, 2003; Saeki $\&$ Saito, 2004a); thus, articulatory suppression impaired performance in the switching block when participants had to maintain both current and future task information to alternately switch tasks. On the basis of these findings, it is assumed that verbal representations are usually used to maintain task information. More specifically, Baddeley et al. proposed that verbal representations underpin the mechanisms for the storage and operation of a taskspecific action control program.

Another paradigm that features a predictable task sequence is the alternating-runs paradigm (Rogers \& Monsell, 1995), in which participants are required to alternate tasks on every second trial in one block of trials (i.e., an AABB ordering of tasks, with A and B representing different tasks). In this paradigm, both switching and repeti-

E. Saeki, e.saeki@hw5.ecs.kyoto-u.ac.jp 
tion trials are included in the same task sequence; consequently, participants must keep track of their position within the sequence, regardless of whether a task switch is required. The difference in performance between switching trials and repetition trials, called the switching cost, reflects the demand of the switching task per se, rather than the demand of maintaining information about task order. Saeki and Saito (2004b) found that RTs were longer and error rates were higher under articulatory suppression conditions than under silent control and foot-tapping conditions in the alternating-runs paradigm, using a format that involved alternating between letter judgments and numerical judgments on every second trial. However, articulatory suppression had equivalent effects on switch trials and nonswitch trials and had no effect on the switching cost. Bryck and Mayr (2005) also examined the effects of articulatory suppression, using an alternating-runs paradigm, and demonstrated results similar to those described above. They suggested that a critical function of verbalization is the endogenous sequencing of tasks on the basis of the sequential characteristics of language. In keeping with earlier arguments presented by Saeki and Saito (2004b), Bryck and Mayr also indicated that articulatory suppression does not interfere with the process of switching between tasks; instead, verbalization plays a critical role in the control of the serial order of tasks.

The finding that the negative effects of articulatory suppression were not confined to switch trials in the alternating-runs paradigm suggests that articulatory suppression in sequential task switching increased the mixing cost, which is the difference in performance between repetition trials within blocks of mixed tasks and single-task trials within pure blocks (Los, 1996; Meiran, Chorev, \& Sapir, 2000). The mixing cost is thought to reflect control processes that are different from those involved in switching tasks, and it has gradually attracted the interest of researchers in this field (Koch, Prinz, \& Allport, 2005; Kray \& Lindenberger, 2000). Rubin and Meiran (2005) systematically examined the nature of mixing costs and suggested that the mixing cost reflects the process of task selection between competitive task sets simultaneously activated in managing multiple task sets. This process is common to repetition and switch trials but is not necessarily involved in single-task blocks.

Note that this task selection process must include or be supported by task order control, at least in predictable/sequential task-switching paradigms such as the alternating-runs paradigm (Bryck \& Mayr, 2005), because it requires reference to task order information. In other words, the task selection process in predictable and sequential task switching might entail a process of sequential task decision based on task order information. That is, participants are required to select the current task set on the basis of the preceding task. This sequential task decision is an integral part of task order control, which is an umbrella term for the endogenous sequencing of tasks and the serial order control of task sets. Verbal representations likely underpin the process of sequential task decision, and articulatory suppression in the predictable and sequential task-switching paradigms might interfere with the sequential task decision, which is required in both switching and repetition trials and should be reflected in the mixing cost. However, whether a sequential task decision is truly supported by verbal representation and is a source of the articulatory suppression effects in the sequential and predictable switching paradigms remains unclear, because the paradigms always include another important aspect of task order control: the retention of task order information. In the alternating-runs paradigm, as well as in the list paradigm, participants perform task blocks, each of which consists of a predetermined task sequence. They must remember the task order, which is provided in advance during experimental instructions, throughout the performance. One may reasonably assume that verbal/phonological working memory supports the retention of task order information because this system is thought to support the retention of serial order. Because the retention of task order and sequential task decision are integrated into task order control and are not separable in the predictable/sequential task-switching paradigms, studies in which those paradigms have been used have so far not been able to clarify whether verbal representations support sequential task decision.

In this article, we expand on results from previous studies and investigate the role of verbal representations in task order control. More specifically, the primary research question here is whether verbal representations can contribute to sequential task decision, or whether such representation just supports the retention of serial order information for tasks in task-switching paradigms. For this purpose, we adopted the random-task-cuing paradigm (Meiran, 1996) with two cue types and examined the effects of articulatory suppression in this paradigm. In a typical task-cuing paradigm, the task order is unpredictable, and a task cue associated with the specific task is presented immediately before each stimulus, informing the participant what the task will be. Thus, participants are not required to identify or retain the task order information. Instead, they are required to switch tasks randomly or to repeat the prior task in response to the task cue. In the present study, in addition to the task cue, we introduced a second type of cue termed a transition cue (Forstmann, Brass, \& Koch, 2007; Forstmann, Brass, Koch, \& von Cramon, 2005; Rushworth, Hadland, Paus, \& Sipila, 2002; Schneider \& Logan, 2007). The transition cue informed participants whether they were to switch or repeat the task but did not indicate the identity of the task itself. Although transition cues are often used to distinguish between the effects of cue transition and those of task transition in task-switching performance (e.g., Forstmann et al., 2007; Schneider \& Logan, 2007), in this study, we focused on a particular characteristic of the transition cue-namely, that it requires sequential task decision. Because a transition cue does not directly indicate a task, participants must refer to task information from a preceding trial to identify the currently relevant task goal, regardless of whether they are required to switch or to repeat the task. Thus, under the transition cue condition, participants are required to select a correct task goal on a trial-by-trial basis. This sequential task decision might not be required under a 
direct-task-cuing condition. If verbal representation is involved in controlling a sequential task decision, the disruption of verbal representation that results from articulatory suppression should reduce performance in both the task-switching and the task repetition trials under the transition-cuing condition. We also required participants to perform pure-task blocks in which they repeated the same task throughout the block. We then directly examined the effects of articulatory suppression on the mixing cost, calculated by subtracting RTs in the pure-task trials from those in the repetition trials. We anticipated that the mixing costs under the transition cue condition would reflect the demands of a sequential task decision, whereas those under the task cue condition would not. Therefore, if articulatory suppression impaired the sequential task decision, the negative effects of articulatory suppression would be observed on the mixing cost under the transition cue condition.

In a series of three experiments, we used a relatively short (e.g., $250 \mathrm{msec}$ ) cue-target interval (CTI), which can be regarded as a preparation interval. Although increasing the preparation interval leads to decreased switch cost in RTs, the findings from a previous study imply that short and long preparation intervals do not have differential effects on articulatory suppression (Saeki et al., 2006). Saeki et al. manipulated the preparation interval by changing the length of the response-stimulus interval (RSI) in the list paradigm and reported that the effects of articulatory suppression on the alternation cost did not differ between the short-RSI $(150 \mathrm{msec})$ and the long-RSI (600 and 1,200 msec) conditions. In consideration of a suggestion that the locus of articulatory suppression on the sequential task switching should be in the mixing cost (Bryck \& Mayr, 2005; Saeki \& Saito, 2004b), the results of Saeki et al. indicate that the length of the preparation interval may not modulate the articulatory suppression effects on the mixing cost in RTs.

Task-switching, task repetition, and pure-task performances were tested under three concurrent task conditions: control (without a secondary task), articulatory suppression, and foot tapping. The primary focus was the effect of articulatory suppression on task performance; the foot-tapping condition was included as a nonspecific dual-task control. Using the random-cuing paradigm, we examined the effects of articulatory suppression with the transition cue in Experiment 1 and with the direct-task cue in Experiment 2. In Experiment 3, to differentiate between the sequential task decision and constant maintenance of task order information during performance, the transition cue and the task cue were presented in the same block, and the effects of articulatory suppression on performance with each cue were examined.

\section{EXPERIMENT 1}

In this experiment, participants were required to perform parity and magnitude judgments for digits, prompted by two types of transition cues: a "+" symbol indicating a task switch and an " $="$ symbol indicating task repetition. In addition to this mixed-task block with the transition cue, participants completed pure-task blocks, in which they performed only one task (i.e., only the parity task or only the magnitude task). Because previous studies had suggested that articulatory suppression increases the mixing cost but not the switch cost (Bryck \& Mayr, 2005; Saeki \& Saito, 2004b), we predicted the same pattern of results if articulatory suppression had an effect on task performance.

\section{Method}

Participants. The participants were 24 undergraduate students from Nihon University.

Apparatus. The experiment was conducted with E-Prime software (Psychology Software Tools, Inc.) running on a computer that displayed stimuli on monitors and registered responses from a serial response box.

Tasks and Stimuli. The stimuli consisted of the digits 1-9, excluding the number 5 . The participants were required to decide whether the digit presented was greater or less than 5 in the magnitude task and whether it was odd or even in the parity task. The task cue was either + , indicating that the participant was to switch tasks, or $=$, indicating that the participant was to repeat the task. In the mixed-task block, a trial started with a 750-msec presentation of two fixation points located above and below the horizontal midline around the center of the display, after which the upper fixation point changed to a cue. At $250 \mathrm{msec}$ after the onset of the cue presentation, a digit stimulus was presented in the lower part of the screen until a response button was pressed, which initiated the next trial and immediately presented the fixation points. The cue was about $10 \mathrm{~mm}$ high and $10 \mathrm{~mm}$ wide. The digit was about $12 \mathrm{~mm}$ high and $8 \mathrm{~mm}$ wide. The cue and digit were displayed in white on a black background, and the distance between the cue and the digit was $5 \mathrm{~mm}$. In the case of the pure block, a meaningless symbol (a "?") was presented at the same position as the transition cue.

The participants responded by pressing the extreme left or the extreme right key on the response box. If the correct response was less than 5 in the magnitude task or odd in the parity task, half the participants were required to press the left key with their left index finger. If the correct response was greater than 5 in the magnitude task or even in the numerical task, they were required to press the right key with their right index finger. The positions of the response keys were reversed for the other participants. If the response was incorrect, an error message ("incorrect") and the name of a task to be performed in the error trial were displayed for $1,500 \mathrm{msec}$.

The participants performed the mixed-and pure-task blocks under three different conditions. Under the silent control condition, the participants completed a task without any dual-task requirements. Under the articulatory suppression condition, they were required to say "da" repeatedly, approximately twice per second, while performing each primary task. Under the foot-tapping condition, the participants were required to tap their left foot at the same pace as that used in the articulatory suppression condition while performing each primary task.

Procedure. The participants sat $60 \mathrm{~cm}$ from the monitor. The experimental session was divided into two phases. In the first phase, the participants completed practice blocks to become familiar with the response keys for each task and the meaning of each cue; each practice block consisted of 24 trials. In response key practice, only the number was presented at the center of the display, and the participants alternated between two response key training blocks for each task. In the practice blocks for the transition cue, the cue and stimulus were displayed one above the other around the center of the monitor (as described above). The participants alternately performed two blocks with the task switch cue $(+)$ and two blocks with the task repetition cue $(=)$. At the end of the practice phase, the participants performed two blocks with randomly changing presentations of the task switch cue and the task repetition cue. If the participants made errors in responding, an error message ("incorrect") and the name of a task to be performed in the error trial were displayed. 
In the subsequent test phase, the participants completed puretask blocks and mixed-task blocks under three concurrent task conditions (control, articulatory suppression, and foot tapping). The test phase was divided into three sessions. The first session consisted of six pure-task blocks, including three magnitude task blocks and three parity task blocks. The participants performed one of the primary two tasks (the magnitude task or the parity task, counterbalanced across participants), completing three successive blocks under control, articulatory suppression, and foot-tapping conditions. They then performed the remaining task (the magnitude or parity task) for three successive blocks, completing one block under each condition. A block in the first session consisted of 24 trials, preceded by 10 practice trials. The secondary task condition had six possible order permutations, and 4 participants were assigned to each order. The order of the secondary task conditions assigned in the first session was adopted in the second and third sessions. At the beginning of both the articulatory suppression and tapping conditions, the participants practiced the relevant secondary task in time to a metronome until they were able to perform the task correctly. In the second session, the participants performed a total of six mixed-task blocks with the transition task cue. A block in this session consisted of 48 trials preceded by 12 practice trials. The participants performed the mixed-task blocks twice under the control, articulatory suppression, and tapping conditions in the same task order as in the first session. In the third session, the participants completed six pure-task blocks, including three magnitude tasks and three parity tasks, under each of three concurrent task conditions. The order of tasks in the third session was the reverse of the order in the first session.

Written instructions for the task to be performed and the relevant condition were given to the participants before each block started, and the experimenter also explained the task and the condition orally. In all blocks, the stimulus and response sequences were pseudorandomized, with a constraint that each task and each stimulus appeared with equal frequency. In a mixed-task block, the repetition trials and the switch trials occurred equally often, with constraints that a task could not be repeated more than twice and that no more than two switches could occur consecutively. The participants were instructed to respond to each task as soon as possible while avoiding errors.

Design. The independent within-participants variables included trial type (repeat and switch) and concurrent task conditions (control, articulatory suppression, and foot tapping).

\section{Results}

Incorrect responses and responses immediately following an error were excluded from the analyses of RT data in all the experiments. Any RT more than three standard deviations from the individual mean for each block under each condition was also excluded. The first trial in each block was not used for any analyses of RTs and errors.

We first will report the results for the direct measure of RTs (the pure blocks and the mixed blocks), then the results on the two types of RT costs (the mixing cost and the switching cost), and finally the results for error rates. Because the error rates were generally low in all three experiments (less than about 5\%) and did not contradict the results for the RTs (i.e., no speed-accuracy trade-off occurred), we will report the mixing and switch costs on the basis of RTs. The mixing cost was calculated by subtracting RTs in the averages of two pure-task blocks from RTs in the repetition trials within the mixed-task block. The switching cost was calculated by subtracting RTs in the repetition trials from RTs in the switch trials within the mixed-task block. For all of the statistical analyses presented in this article, we used an alpha level of .05.
Table 1A

Mean Reaction Times (in Milliseconds; With Standard Errors) for Different Tasks in Each Condition in Experiment 1

\begin{tabular}{|c|c|c|c|c|c|c|c|c|}
\hline \multirow[b]{3}{*}{ Condition } & \multicolumn{4}{|c|}{ Pure Block } & \multicolumn{4}{|c|}{ Mixed Block } \\
\hline & \multicolumn{2}{|c|}{ Parity } & \multicolumn{2}{|c|}{ Magnitude } & \multicolumn{2}{|c|}{ Repetition } & \multicolumn{2}{|c|}{ Switch } \\
\hline & $M$ & $S E$ & $M$ & $S E$ & $M$ & $S E$ & $M$ & $S E$ \\
\hline Control & 510 & 14 & 423 & 8 & 931 & 40 & 1,132 & 49 \\
\hline Articulation & 524 & 12 & 451 & 12 & 1,197 & 82 & 1,397 & 89 \\
\hline Tapping & 521 & 14 & 462 & 11 & 1,022 & 55 & 1,223 & 66 \\
\hline
\end{tabular}

RTs. The left part of Table 1A shows the mean RTs from the pure-task blocks as a function of the primary task (parity and magnitude) and concurrent task condition (control, articulatory suppression, and foot tapping). The RTs in the pure-task blocks were submitted to a primary task $\times$ concurrent task repeated measures ANOVA. The main effect of the primary task was significant $[F(1,23)=$ $\left.68.12, M S_{\mathrm{e}}=2,782.89\right]$, and the RTs in the parity task were longer than those in the magnitude task. The main effect of concurrent task was significant $[F(2,46)=5.33$, $\left.M S_{\mathrm{e}}=1,630.25\right]$. A marginally significant interaction between primary task and concurrent task condition was also found $\left[F(2,46)=3.05, M S_{\mathrm{e}}=733.60, p=.06\right]$, indicating that only in the magnitude task did RTs tend to be slower under the articulatory and tapping conditions, as compared with the control condition.

The right part of Table 1A shows the mean RTs from the mixed-task block as a function of trial type (repeat and switch) and concurrent task condition. A two-way ANOVA revealed a significant main effect of trial type $\left[F(1,23)=54.17, M S_{\mathrm{e}}=26,873.83\right]$, indicating that RTs in the switch trials were longer than those in the repetition trials under all conditions. The main effect of concurrent task condition was also significant $[F(2,46)=21.79$, $\left.M S_{\mathrm{e}}=40,106.74\right]$. RTs under the articulatory suppression condition were longer than those under the tapping and control conditions. RTs in the tapping condition were longer than those in the control condition. No significant interaction was found between trial type and concurrent task factors $(F<1)$.

RT costs. Figure 1 shows mixing cost and switching cost as a function of concurrent task. A one-way ANOVA on the mixing cost revealed a significant main effect of concurrent task $\left[F(2,46)=15.03, M S_{\mathrm{e}}=25,645.84\right]$, indicating that articulatory suppression led to an increase in the mixing cost, as compared with tapping and the control. No significant difference was found between the mixing cost under tapping and that under control conditions ( $p=$ .15). The switch costs, however, were almost equal among the three concurrent task conditions $(F<1)$.

Errors. The left part of Table 1B shows the mean error rates from the pure blocks as a function of primary task and concurrent task factors. A two-way ANOVA revealed a significant main effect of primary task $[F(1,23)=17.02$, $\left.M S_{\mathrm{e}}=0.00062\right]$. More errors were observed in the parity task than in the magnitude task. A main effect of concurrent task condition was also significant $\left[F(2,46)=4.43, M S_{\mathrm{e}}=\right.$ $0.00035]$, indicating that articulatory suppression and tapping $(p=.09)$ resulted in increased errors, as compared with 


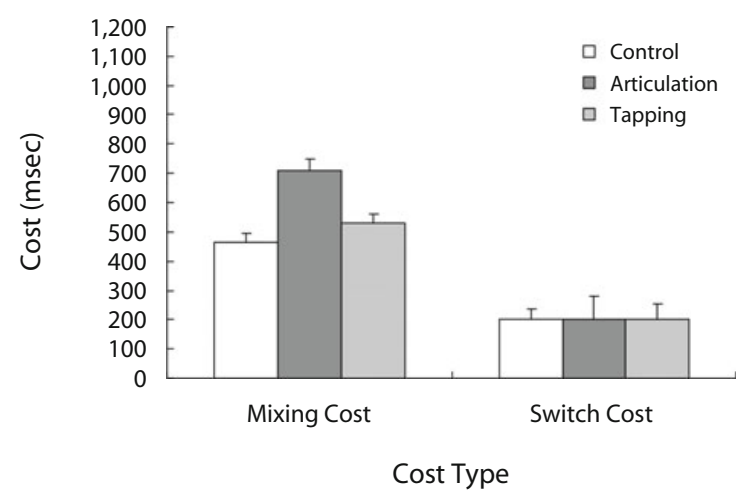

Figure 1. Mean mixing costs (left) and switch costs (right) in control, articulatory suppression, and tapping conditions in Experiment 1 . The bars represent the standard errors.

the control condition. No interaction was found between the primary task and concurrent task factors $(F<1)$.

The right part of Table 1B also shows the mean error rates from the mixed block as a function of trial type and concurrent task. A two-way ANOVA revealed significant main effects of trial type $\left[F(1,23)=7.35, M S_{\mathrm{e}}=0.00099\right]$ and of concurrent task $\left[F(2,46)=11.71, M S_{\mathrm{e}}=0.00087\right]$. More errors occurred in the switch trials than in the repetition trials, and errors under articulatory suppression exceeded those under other concurrent task conditions. The interaction between trial type and concurrent task was not significant $(F<1)$.

\section{Discussion}

Articulatory suppression increased RTs as well as errors in both the repetition and the switch trials, as compared with the tapping and control conditions. The articulatory suppression effects in the repetition trials were comparable in size to those in the switch trials. Consequently, articulatory suppression had no significant effects on the switch costs. Furthermore, articulatory suppression had no specific effects on responses in the pure blocks in which task order control was not required. Accordingly, the mixing costs were larger under the suppression condition than under the control and tapping conditions. The finding that articulatory suppression did not affect the switch costs is consistent with data from previous studies in which predictable and sequential task switching was used (Bryck \& Mayr, 2005; Saeki \& Saito, 2004b) and suggests that articulatory suppression did not specifically disturb the switching task, at least in this (short-CTI) random-cuing paradigm. More important, when the participants were required to refer to task information from previous trials in selecting the current task set, articulatory suppression impaired task performance, suggesting that verbal representation is involved in transient task order control- that is, in the sequential task decision.

However, an alternative interpretation of the effect of articulatory suppression in the mixed blocks with the transition cue is also possible. Although the sequential task decision could be essential for performance of the mixed block, the participants were also required to decode the meaning of the cue in mixed blocks (Logan \& Schneider, 2006), whereas the cue-decoding process was not mandatory in the pure-task blocks. In fact, Miyake, Emerson, Padilla, and Ahn (2004) reported the effects of articulatory suppression on the mixing and switching costs in a random-cuing paradigm with a letter cue that provided the first letter of the current task name, thus indicating the identity of the task. Therefore, it remains possible that articulatory suppression might affect the process of decoding a cue, rather than the process of making a sequential task decision. In the next experiment, we examined the effects of articulatory suppression, using a direct-task cue instead of a transition cue. If the cue-decoding process contributes to the articulatory suppression effect observed in Experiment 1, the effect on the mixing costs should occur even with the direct-task cues.

\section{EXPERIMENT 2}

In this experiment, we adopted the symbols used in Experiment 1 (i.e., + and $=$ ) as the task cues. However, although the + and $=$ symbols indicated that the participants were to switch or repeat the task, respectively, in Experiment 1, these symbols in Experiment 2 were directly associated with the parity and magnitude tasks, respectively. Because the symbol-task match is arbitrary and not a previously learned association, the task cue in Experiment 2 could not automatically provide task information. Therefore, the participants had first to decode the task cues to identify the task to be performed in the current trial. Note that the direct-task cue did not require a sequential task decision; that is, the participants did not need to refer to task information from the preceding trial to identify the current task. If the negative effects of articulatory suppression in Experiment 1 were due to impairment of the cue-decoding process, we would expect to observe those same effects in this experiment.

Table 1B

Mean Error Rates (With Standard Errors) for Different Tasks in Each Condition in Experiment 1

\begin{tabular}{|c|c|c|c|c|c|c|c|c|}
\hline \multirow[b]{3}{*}{ Condition } & \multicolumn{4}{|c|}{ Pure Block } & \multicolumn{4}{|c|}{ Mixed Block } \\
\hline & \multicolumn{2}{|c|}{ Parity } & \multicolumn{2}{|c|}{ Magnitude } & \multicolumn{2}{|c|}{ Repetition } & \multicolumn{2}{|c|}{ Switch } \\
\hline & $M$ & $S E$ & $M$ & $S E$ & $M$ & $S E$ & $M$ & $S E$ \\
\hline Control & .025 & .005 & .004 & .002 & .033 & .007 & .047 & .008 \\
\hline Articulation & .035 & .007 & .017 & .005 & .063 & .008 & .073 & .010 \\
\hline Tapping & .023 & .005 & .011 & .003 & .037 & .005 & .056 & .007 \\
\hline
\end{tabular}


Table 2A

Mean Reaction Times (in Milliseconds; With Standard Errors) for Different Tasks in Each Condition in Experiment 2

\begin{tabular}{|c|c|c|c|c|c|c|c|c|}
\hline \multirow[b]{3}{*}{ Condition } & \multicolumn{4}{|c|}{ Pure Block } & \multicolumn{4}{|c|}{ Mixed Block } \\
\hline & \multicolumn{2}{|c|}{ Parity } & \multicolumn{2}{|c|}{ Magnitude } & \multicolumn{2}{|c|}{ Repetition } & \multicolumn{2}{|c|}{ Switch } \\
\hline & $M$ & $\overline{S E}$ & $M$ & $S E$ & $M$ & $S E$ & $M$ & $S E$ \\
\hline Control & 519 & 14 & 460 & 11 & 644 & 24 & 724 & 33 \\
\hline Articulation & 547 & 14 & 48 & 12 & 710 & 33 & 779 & 39 \\
\hline Tapping & 551 & 14 & 511 & 12 & 693 & 39 & 755 & \\
\hline
\end{tabular}

\section{Method}

The method and procedures in this experiment were identical to those used in Experiment 1, except that a cue in the mixed-task block directly indicated the task to be performed in the current trial. If the + sign was presented, the participants were required to perform the magnitude task. If the = sign was presented, they were required to perform the parity task. Twenty-four students from Kyoto University participated in this experiment.

\section{Results}

The results from Experiment 2 were analyzed and will be reported in the same manner as those in Experiment 1.

RTs. The left part of Table 2A shows the mean RTs from the pure-task blocks as a function of primary task and concurrent task condition. A two-way ANOVA for these data revealed a significant main effect of primary task $\left[F(1,23)=30.10, M S_{\mathrm{e}}=3,285.79\right]$, indicating that the RTs in the parity task were longer than those in the magnitude task. A main effect of concurrent task was also significant $\left[F(2,46)=8.26, M S_{\mathrm{e}}=2,591.52\right]$, indicating that tapping prolonged RTs, as compared with the control condition. No significant interaction was found between the primary task and concurrent task factors $[F(2,46)=$ 2.18, $\left.M S_{\mathrm{e}}=631.47, p=.12\right]$.

The right part of Table 2A shows the mean RTs from the mixed block as a function of trial type and concurrent task. A two-way ANOVA for these data revealed a significant main effect of trial type $\left[F(1,23)=36.91, M S_{\mathrm{e}}=\right.$ $4,821.94]$, indicating that RTs in the switch trials were longer than those in the repetition trials. A main effect of concurrent task was also significant $[F(2,46)=3.83$, $\left.M S_{\mathrm{e}}=11,757.76\right]$. Although the RTs under the articulatory suppression condition were longer than those under the control condition, RTs under the articulatory suppression and tapping conditions did not differ significantly $(p=1)$. No significant interaction was found between the trial type and concurrent task factors $(F<1)$.

RT costs. Figure 2 shows the RT mixing costs and switch costs as a function of the concurrent task condition. Main effects of concurrent task were not significant for the mixing $\operatorname{cost}\left[F(2,46)=1.30, M S_{\mathrm{e}}=7,686.12, p=\right.$ $.28]$ or for the switch cost $(F<1)$.

Errors. The left part of Table 2B shows the mean error rates from the pure-task block. A two-way ANOVA revealed a significant main effect of primary task $[F(1,23)=$ $\left.18.70, M S_{\mathrm{e}}=0.00045\right]$. More errors were observed in the parity task than in the magnitude task. The main effect of the concurrent task condition $\left[F(2,46)=1.37, M S_{\mathrm{e}}=\right.$ $0.00024, p=.26]$ and the interaction between task and condition $(F<1)$ were not significant.
The right part of Table 2B shows the mean error rates from the mixed blocks as a function of trial type and concurrent task condition. A two-way ANOVA revealed a significant main effect of trial type $\left[F(1,23)=8.80, M S_{\mathrm{e}}=\right.$ $0.00016]$. More errors were observed in the switch trials than in the repetition trials. The main effect of concurrent task $\left[F(2,46)=2.31, M S_{\mathrm{e}}=0.00075, p=.11\right]$ and the interaction between trial type and concurrent task condition $\left[F(2,46)=1.27, M S_{\mathrm{e}}=0.00081, p=.29\right]$ were not significant.

\section{Discussion}

The aim of Experiment 2 was to examine whether articulatory suppression impairs the cue-decoding processes. Although the participants had to decode the arbitrary symbol cues to obtain task information for the current trial, articulatory suppression did not have specific and distinct effects beyond the effects of concurrent task demands in this experiment. Thus, the effects of articulatory suppression obtained in Experiment 1 were most likely due to the requirement of a sequential task decision posed by the transition cue, rather than to the requirement for cue decoding. To examine the effects of the two types of cues directly, we performed a combined analysis on the mixing costs, with experiment as a between-participants variable and concurrent task condition as a within-participants variable. The focus of the analysis was on the interaction between the experiment and concurrent task conditions. A two-way ANOVA showed a significant interaction $\left[F(2,92)=8.16, M S_{\mathrm{e}}=16,666.02\right]$, indicating that articulatory suppression affected the mixing costs differentially for the two types of cue.

Some discrepancy is apparent between the results in this experiment and those of Miyake et al. (2004). The latter reported that when the letter cue (the first letter of the task name) was provided in random-task cuing, articulatory suppression prolonged RTs in the switch trials, as compared with those in the repetition trials, resulting in an increased switch cost. Also, articulatory suppression prolonged RTs in the repetition trials, as compared with those in the pure-task trials in their study, resulting in increased

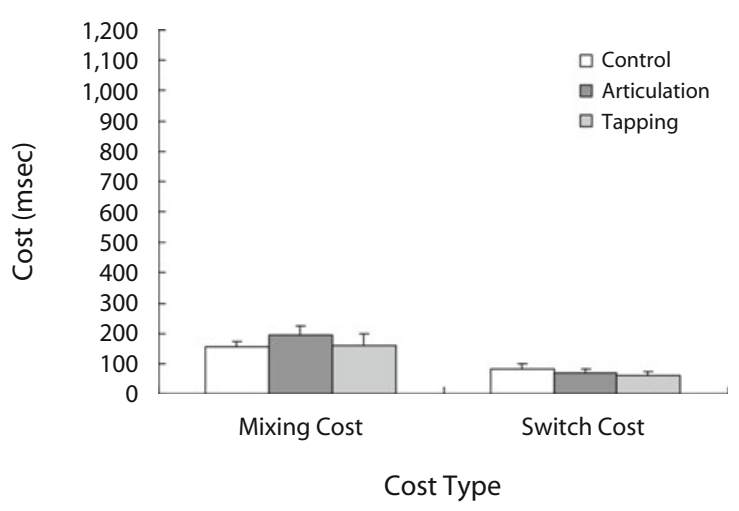

Figure 2. Mean mixing costs (left) and switch costs (right) in control, articulatory suppression, and tapping conditions in Experiment 2 . The bars represent the standard errors. 
Table 2B

Mean Error Rates (With Standard Errors) for Different Tasks

in Each Condition in Experiment 2

\begin{tabular}{|c|c|c|c|c|c|c|c|c|}
\hline \multirow[b]{3}{*}{ Condition } & \multicolumn{4}{|c|}{ Pure Block } & \multicolumn{4}{|c|}{ Mixed Block } \\
\hline & \multicolumn{2}{|c|}{ Parity } & \multicolumn{2}{|c|}{ Magnitude } & \multicolumn{2}{|c|}{ Repetition } & \multicolumn{2}{|c|}{ Switch } \\
\hline & $M$ & $S E$ & $M$ & $S E$ & $M$ & $S E$ & $M$ & $S E$ \\
\hline Control & .020 & .006 & .005 & .002 & .016 & .005 & .044 & .007 \\
\hline Articulation & .027 & .006 & .009 & .003 & .037 & .006 & .047 & .011 \\
\hline Tapping & .022 & .005 & .009 & .003 & .028 & .006 & .050 & .009 \\
\hline
\end{tabular}

mixing cost. In contrast, we found no suppression effects on the switch cost and the mixing cost in this experiment, using + and $=$ symbols as direct-task cues. One apparent difference between the two studies is the length of the CTI. Whereas we used a short CTI $(250 \mathrm{msec})$, Miyake et al. used a long CTI $(1,200 \mathrm{msec})$ and gave their participants opportunities for advance preparation prior to the next trial. They reported that the switching cost in articulatory suppression under the long-CTI condition was almost equivalent to that in silent control (without any dual-task requirement) under the short-CTI $(200 \mathrm{msec})$ condition, implying that articulatory suppression might interfere with the advance preparation processes in random-task cuing with a task cue (see also Goschke, 2000). How much the length of the CTI influences the effect of articulatory suppression in random-task cuing with a direct-task cue remains unclear because no study so far has directly compared effects with a long CTI with those with a short CTI. However, the negative effects of articulatory suppression on the switch cost may become more pronounced with a long CTI.

Note that the first and second experiments in the present study differed not only in the presence/absence of sequential task decision, but also in the degree of the potential general memory load. In Experiment 1, the participants might have tried to remember the identity of every previous task during performance of the mixed block because they knew that such information was essential for the current task. In Experiment 2, however, the participants were asked simply to follow the direct-task cue to perform the current tasks, and thus maintenance of prior task information was not required. Therefore, the possibility remains that the difference in memory requirements might have led to the differential effects of articulatory suppression in the two experiments. In Experiment 3, we examined the effect of articulatory suppression on transition and directtask cues, again using the random-task-cuing paradigm with a secure control of memory demand.

\section{EXPERIMENT 3}

In this experiment, the transition and direct-task cues were presented randomly within the same block. Because the participants were uncertain whether the next trial would consist of the transition or the direct-task cue, they might have tried to remember the nature of the previous task for all trials; alternatively, they might not have tried to remember such information in any of the trials. We had no reason to assume that the participants would use dif- ferential task strategies for the transition cues and for the direct-task cues in this experiment. Therefore, a reasonable prediction was that articulatory suppression would have identical effects on the transition and the task cues, given that differential task strategies (e.g., attempts to remember previous task information) led to the differential results in Experiments 1 and 2.

For this study, we prepared four cues: two transition cues and two direct-task cues. For Experiments 1 and 2, employing the same cues $(+$ and $=$ symbols $)$ was important to ensure identical procedures in the two experiments, except with regard to the meaning of those cues. However, one might argue that the + symbol implicitly indicates a change, whereas the $=$ symbol suggests a repetition. These potential semantic associations might have affected task performance when these symbols were used as transition cues in Experiment 1, but not when they were used as task cues in Experiment 2. Therefore, in this experiment, we used semantically neutral symbols (! and \#) for transition cues, whereas + and $=$ symbols were used for direct-task cues.

\section{Method}

Participants. The participants were 24 students from Kyoto University and Nihon University.

Tasks and Stimuli. The tasks and stimuli were identical to those in Experiments 1 and 2, except that four symbols $(+,=, !$, and \#) were used as cues in this experiment. The + and $=$ signs were used as task cues; the + sign preceded a magnitude task, and the $=$ sign preceded a parity task. The ! and \# signs were used as transition cues; the ! sign indicated that the participant was to repeat the task performed in the preceding trial, and the \# sign meant that the participant was to switch to a different task.

Procedure. The procedures were similar to those in Experiments 1 and 2, except for the following points. In the mixed-task blocks, which consisted of 96 trials preceded by 12 practice trials, the two task cues and the two transition cues were presented randomly but with equal frequency. Repetition trials and switch trials also occurred with equal frequency. In the middle of the mixed block, a rest phase was introduced to reduce fatigue from this prolonged dual-task experiment. Practice blocks for learning the cues were conducted just before the mixed-task blocks. In this cue-learning session, the participants alternately performed the transition cue blocks and the task cue blocks three times and then practiced two blocks in which the transition cues and the task cues were presented randomly. The block for learning the meaning of each cue consisted of 24 trials, and the practice block presenting all cues consisted of 48 trials.

As in Experiments 1 and 2, the test session consisted of six puretask blocks (including three magnitude task and three parity task blocks), six mixed-task blocks (plus the cue-learning blocks in advance), and six pure-task blocks (including three magnitude task and three parity task blocks). The participants completed each task block 
Table 3A

Mean Reaction Times (in Milliseconds; With Standard Errors) for Different Tasks in Each Condition in Experiment 3

\begin{tabular}{|c|c|c|c|c|c|c|c|c|c|c|c|c|}
\hline \multirow[b]{4}{*}{ Condition } & & & & & \multicolumn{8}{|c|}{ Mixed Block } \\
\hline & \multicolumn{4}{|c|}{ Pure Block } & \multicolumn{4}{|c|}{ Transition Cue } & \multicolumn{4}{|c|}{ Task Cue } \\
\hline & \multicolumn{2}{|c|}{ Parity } & \multicolumn{2}{|c|}{ Magnitude } & \multicolumn{2}{|c|}{ Repetition } & \multicolumn{2}{|c|}{ Switch } & \multicolumn{2}{|c|}{ Repetition } & \multicolumn{2}{|c|}{ Switch } \\
\hline & $M$ & $S E$ & $M$ & $S E$ & $M$ & $S E$ & $M$ & $S E$ & $M$ & $S E$ & $M$ & $S E$ \\
\hline Control & 528 & 25 & 445 & 14 & 1,160 & 70 & 1,318 & 64 & 860 & 34 & 985 & 60 \\
\hline Articulation & 510 & 16 & 470 & 20 & 1,376 & 98 & 1,524 & 94 & 959 & 52 & 1,052 & 65 \\
\hline Tapping & 541 & 19 & 481 & 19 & 1,180 & 64 & 1,331 & 90 & 938 & 57 & 1,080 & 70 \\
\hline
\end{tabular}

under each concurrent task condition (i.e., silent control, articulatory suppression, and foot tapping). During the tasks, the participants were required to press the designated response key as soon as possible, while avoiding errors.

Design. The independent within-participants variables were cue type (transition or direct-task cues), trial type (repeat and switch), and concurrent task condition (control, articulatory suppression, and foot tapping).

\section{Results}

The trials immediately following the brief rest were excluded, in addition to the trials excluded on the basis of the same criteria as those in Experiments 1 and 2.

RTs. The left part of Table 3A shows RTs from the pure-task blocks as a function of primary task and concurrent task. As the table shows, no significant effect of concurrent task was observed $\left[F(2,46)=2.03, M S_{\mathrm{e}}=\right.$ $4,149.23, p=.14]$, although RTs were shorter in the magnitude task than in the parity task under all three conditions $\left[F(1,23)=29.66, M S_{\mathrm{e}}=4,563.95\right]$.

The right part of Table 3A shows RTs from the mixed blocks as a function of cue type, trial type, and concurrent task. A three-way ANOVA revealed significant main effects of all independent variables [cue type, $F(1,23)=$ $102.59, M S_{\mathrm{e}}=78,975.86$; trial type, $F(1,23)=32.74$, $M S_{\mathrm{e}}=40,933.09$; concurrent task, $F(2,46)=9.50$, $\left.M S_{\mathrm{e}}=56,323.65\right]$. The RTs in the switch trials were longer than those in the repetition trials, regardless of cue type and concurrent task. The RTs in trials with transition cues were also longer than those with task cues under all concurrent task conditions. A significant interaction was found between cue type and concurrent task $[F(2,46)=$ $\left.18.57, M S_{\mathrm{e}}=12,992.55\right]$. Specific effects of articulatory suppression were observed in trials with transition cues $\left[F(2,46)=15.87, M S_{\mathrm{e}}=28,053.07\right]$, and articulatory suppression led to increased RTs, as compared with the tapping and control conditions. In trials with task cues $\left[F(2,46)=4.16, M S_{\mathrm{e}}=13,805.03\right], \mathrm{RT}$ s in the control condition were shorter than those under the tapping and articulatory suppression $(p=.09)$ conditions.

RT costs. Figure 3 shows the RT mixing costs and switch costs as a function of cue type and the concurrent task. A two-way ANOVA on the mixing costs revealed a significant main effect of cue type $[F(1,23)=49.50$, $\left.M S_{\mathrm{e}}=73,463.01\right]$. Under all concurrent task conditions, the mixing costs with a transition cue were larger than those with the task cue. A main effect of the concurrent task was also significant $\left[F(2,46)=7.32, M S_{\mathrm{e}}=44,876.37\right]$. A significant interaction between cue type and the concur- rent task was found $\left[F(2,46)=3.18, M S_{\mathrm{e}}=29,899.14\right]$. The mixing costs with the transition cue were larger under the articulatory suppression condition than under the tapping and control conditions. The mixing costs with the task cue under the suppression condition, however, tended to be larger than those under the control condition ( $p=.08)$, but not greater than those under the tapping condition $(p=.28)$.

For the switch cost, an ANOVA detected no significant effects (all $\left.F_{\mathrm{S}}<1\right)$.

Errors. The left part of Table 3B shows the mean error rates from the pure-task blocks as a function of the primary task and concurrent task. A two-way ANOVA revealed a significant main effect of the primary task $\left[F(1,23)=16.14, M S_{\mathrm{e}}=0.00033\right]$, indicating that error rates were higher in the parity task than in the magnitude task. The main effect of the concurrent task $(F<1)$ and the interaction between trial type and the concurrent task condition $\left[F(2,46)=1.63, M S_{\mathrm{e}}=0.00021, p=.21\right]$ were not significant.

The right part of Table 3B shows the mean error rates from the mixed blocks as a function of the concurrent task and trial type in each cue type. A three-way ANOVA revealed a significant main effect of cue type $[F(1,23)=$ $\left.26.39, M S_{\mathrm{e}}=0.00091\right]$, indicating that more errors occurred in trials with transition cues than in those with task cues. A main effect of trial type was also significant $\left[F(1,23)=9.55, M S_{\mathrm{e}}=0.00061\right]$. More errors were ob-

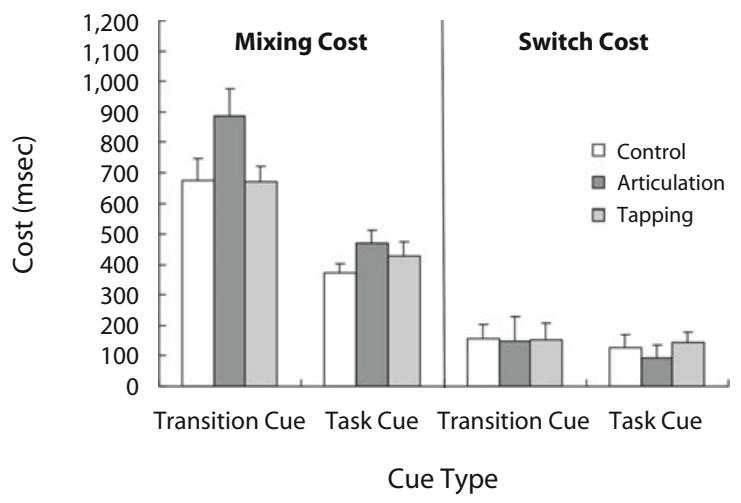

Figure 3. Mean mixing costs (left) and switch costs (right) for the transition cue and the task cue in control, articulatory suppression, and tapping conditions in Experiment 3. The bars represent the standard errors. 
Table 3B

Mean Error Rates (With Standard Errors) for Different Tasks

in Each Condition in Experiment 3

\begin{tabular}{|c|c|c|c|c|c|c|c|c|c|c|c|c|}
\hline \multirow[b]{4}{*}{ Condition } & & & & & \multicolumn{8}{|c|}{ Mixed Block } \\
\hline & \multicolumn{4}{|c|}{ Pure Block } & \multicolumn{4}{|c|}{ Transition Cue } & \multicolumn{4}{|c|}{ Task Cue } \\
\hline & \multicolumn{2}{|c|}{ Parity } & \multicolumn{2}{|c|}{ Magnitude } & \multicolumn{2}{|c|}{ Repetition } & \multicolumn{2}{|c|}{ Switch } & \multicolumn{2}{|c|}{ Repetition } & \multicolumn{2}{|c|}{ Switch } \\
\hline & $M$ & $\overline{S E}$ & $M$ & $S E$ & $M$ & $S E$ & $M$ & $S E$ & $M$ & $S E$ & $M$ & $S E$ \\
\hline Control & .021 & .004 & .005 & .002 & .024 & .006 & .037 & .006 & .015 & .004 & .013 & .003 \\
\hline Articulation & .017 & .003 & .011 & .003 & .038 & .008 & .050 & .009 & .014 & .006 & .029 & .007 \\
\hline Tapping & .023 & .004 & .008 & .002 & .033 & .007 & .038 & .009 & .016 & .004 & .026 & .006 \\
\hline
\end{tabular}

served in the switch trials than in the repetition trials. No main effect of condition was detected $[F(2,46)=2.21$, $\left.M S_{\mathrm{e}}=0.00119, p=.12\right]$, and no interaction between independent variables was observed $(F \mathrm{~s}<1)$.

\section{Discussion}

The results of this experiment in the transient cue trials replicated those in Experiment 1, confirming that the mixing costs that reflect serial task decision increased under the articulatory suppression condition, as compared with the tapping and control conditions. The mixing costs for trials with the task cue, however, tended to be larger under articulatory suppression, as compared with the control condition, whereas no such tendency was found in Experiment 2 . Because the difference in the mixing costs between the articulatory suppression and tapping conditions for the task cue trials did not reach significance, it seems reasonable to suppose that articulatory suppression did not have a specific effect beyond concurrent task demands. Perhaps the requirement to remember four cues increased the task difficulty and made the participants more susceptible to dual-task demand. It is also possible, however, that this significant trend is genuine - that is, that articulatory suppression leads to longer RTs than does the control condition, even for task cue trials in this experiment. This suggests that articulatory suppression affected the mixing costs through the disruption of memory for task order. Even accepting this argument, we can safely state that articulatory suppression had a larger impact on the mixing costs for the transition cue trials than for the task cue trials and that verbal representations play an important role in the sequential task decision beyond simply the retention of task order.

Therefore, the results of Experiment 3 rule out the possibility that the differential patterns of results in Experiments 1 and 2 were entirely caused by a difference in participants' task strategies in each experiment, such as remembering previous task information and scheduling tasks between the main task and the concurrent task (articulatory suppression and tapping). In Experiment 3, both the transition cue and the task cue were presented unpredictably in the same block, and it would have been difficult or even impossible for the participants to employ different strategies for each type of cue.

The findings in Experiment 3, in which the general memory demand, the cue-decoding process, and the task strategy were shared between the transition and task cues, suggest that the primary locus of the articulatory suppres- sion effect in this study may have been in the transient task order control - that is, in the sequential task decision.

\section{GENERAL DISCUSSION}

The present study was designed to clarify the role of verbal representations in task order control in a taskswitching paradigm. In previous studies in which predictable and sequential task switching was used (Baddeley et al., 2001; Bryck \& Mayr, 2005; Emerson \& Miyake, 2003; Saeki \& Saito, 2004a, 2004b; Saeki et al., 2006), it was difficult to verify the involvement of verbal representations in the management of sequential task decision during performance. This difficulty derives from the fact that both the list paradigm and the alternating-runs paradigm require participants to remember the task sequence prior to performing task blocks. We used the random-task-cuing paradigm with two different types of cues to examine the involvement of verbal representations in a sequential task decision.

When a cue conveyed only information about task transition (Experiment 1), articulatory suppression impaired performance regardless of trial type (repeat and switch), resulting in an increase in the mixing cost, but not in the switch cost. In contrast, the results of Experiment 2 showed no specific effect of articulatory suppression when a cue was associated directly with a task. These results eliminate the possibility that the negative effect of articulatory suppression in Experiment 1 was due to interference with cue-decoding processes, which are necessary in task-cuing paradigms. The results of Experiment 3 also ruled out the explanation of the suppression effects based solely on general memory demand and task strategy. The negative effect of articulatory suppression was observed only in trials with transition cues, even if the transition cue and the task cue were presented in the same block. The results from this series of three experiments indicate that the use of verbal representations was effective in selecting a task when the participants were asked to determine a task through reference to the preceding task.

The general pattern of the results in the present study is perfectly compatible with data from a recent fMRI study by Forstmann et al. (2005), who presented the transition cue and the task cue within the same block and examined the difference between brain activations in transition cue trials and those in task cue trials. Their results revealed several activation foci along the left inferior frontal sulcus in the transition cue trials and, directly relevant to our 
study, left-side activation in the posterior part of the inferior frontal gyrus (BA44). The activations were observed in both repetition and switch trials when the transition cue was presented, but not when the direct-task cue was given. Considering that the activation area corresponds to Broca's area, which is closely connected to verbal/language function (Dominey, Hoen, Blanc, \& Lelekov-Boissard, 2003), these results suggest that verbal function was implicated in the trials involving the transient task cue.

Note that the finding in this study does not imply that verbal representation has nothing to do with the directtask set retrieval from a task cue. The use of a short CTI clearly limits interpretations of our data. Miyake et al. (2004) reported that articulatory suppression had effects on performance in a task cue situation using a long preparation interval and verbal cues. As was noted in the Discussion section for Experiment 2, it seems that the length of the CTI is an important factor that might modulate the effect of articulatory suppression in task cue trials because verbal representations might operate during the preparation interval in switching trials (e.g., Goschke, 2000; Miyake et al., 2004). Our results are restricted to situations in which symbolic/arbitrary task cues are employed with a short CTI. Therefore, we cannot apply our data beyond this limitation, and it is still possible to argue that verbal representation might be involved in the direct-task set retrieval from a task cue in some situations, such as with a long preparation interval and/or using a verbal task cue (e.g., Miyake et al., 2004).

Notwithstanding its limitations, the results of this study suggest the involvement of verbal representation under transition cue conditions. What then would be the role of verbal representations in the random-cuing paradigm with transition cues? In this study, articulatory suppression had specific negative effects on the mixing cost, but not on the switch cost. These results indicate that the role of verbal representations in transition cue trials may be related to possible sources of the mixing costs. In mixed-task blocks, the target stimuli were bivalent (i.e., overlapping between the two tasks) and could activate both correct and incorrect responses, resulting in competition between the two tasks in the switching and the repetition trials. Rubin and Meiran (2005) suggested that the mixing cost reflects a control process that resolves task competition triggered by a bivalent target stimulus in a mixed-task block, regardless of trial type. They assume that the transient control process, such as a task decision, could resolve bottom-up interference caused by stimulus ambiguity. The effects of articulatory suppression on the mixing cost reported in the present study support the idea that verbal representations contribute to transient task decision processes in the resolution of bottom-up interference. However, the finding that the negative effects of articulatory suppression on mixing costs was not observed in the task cue trials indicates that verbal representation is not necessarily required in task decision processes. Verbal representations might be useful for task decision processes only when endogenous control is strongly required due to the absence of external support that identifies the current task - as under the transition cue condition, the situation in which a se- quential task decision was required. Schneider and Logan (2007) offered a related suggestion that a transition cue requires combining the meaning of the cue (i.e., repeat or switch) with knowledge of the task performed in the preceding trial. They assumed that participants used this combination to retrieve a task name mediator. According to this mediator retrieval hypothesis, nontransparent cues demand that the participants retrieve a mediator, such as a task name, to select a correct response (Logan \& Bundesen, 2004; Logan \& Schneider, 2006). Perhaps articulatory suppression interferes with the retrieval of a task name mediator, which may be in a verbal format (see Logan \& Schneider, 2006).

The two explanations above are not exclusive in the sense that they handle mechanisms at different levels. It might be possible that verbal representations operate in both/either retrieving a task name mediator and/or resolving bottom-up competition. Although the precise mechanisms are still to be explored, we were able to reach the following conclusion through this series of three experiments: The use of verbal representation is effective in selecting a task set on the basis of transient task order information in both repetition and switch trials; in other words, it is effective in driving action using information from the immediate past.

\section{AUTHOR NOTE}

This research was partly supported by grants from the Japan Society for the Promotion of Science to E.S. Correspondence concerning this article should be addressed to E. Saeki, Department of Cognitive Psychology in Education, Graduate School of Education, Kyoto University, Yoshida-Hommachi, Kyoto 606-8501, Japan (e-mail: e.saeki@hw5.ecs .kyoto-u.ac.jp).

\section{REFERENCES}

Allport, A., Styles, E. A., \& Hsieh, S. (1994). Shifting intentional set: Exploring the dynamic control of tasks. In C. Umiltà \& M. Moscovitch (Eds.), Attention and performance XV: Conscious and nonconscious information processing (pp. 421-452). Cambridge, MA: MIT Press, Bradford Books.

Baddeley, A. D., Chincotta, D., \& Adlam, A. (2001). Working memory and the control of action: Evidence from task switching. Journal of Experimental Psychology: General, 130, 641-657.

BRYCK, R. L., \& MAYR, U. (2005). On the role of verbalization during task set selection: Switching or serial order control? Memory \& Cognition, 33, 611-623.

Dominey, P. F., Hoen, M., Blanc, J. M., \& Lelekov-Boissard, T. (2003). Neurological basis of language and sequential cognition: Evidence from simulation, aphasia, and ERP studies. Brain \& Language, 86, 207-225.

Emerson, M. J., \& MiYAKe, A. (2003). The role of inner speech in task switching: A dual-task investigation. Journal of Memory \& Language, 48, 148-168.

Forstmann, B. U., Brass, M., \& Koch, I. (2007). Methodological and empirical issues when dissociating cue-related from task-related processes in the explicit task-cuing procedure. Psychological Research, 71, 393-400.

Forstmann, B. U., Brass, M., Koch, I., \& von Cramon, D. Y. (2005). Internally generated and directly cued task sets: An investigation with fMRI. Neuropsychologia, 43, 943-952.

GoschKe, T. (2000). Intentional reconfiguration and involuntary persistence in task set switching. In S. Monsell \& J. Driver (Eds.), Control of cognitive processes: Attention and performance XVIII (pp. 331-355). Cambridge, MA: MIT Press.

Jersild, A. T. (1927). Mental set and shift. Archives of Psychology (Whole No. 89). 
Koch, I., Prinz, W., \& Allport, A. (2005). Involuntary retrieval in alphabet-arithmetic tasks: Task-mixing and task-switching costs. Psychological Research, 69, 252-261.

Kray, J., \& Lindenberger, U. (2000). Adult age differences in task switching. Psychology \& Aging, 15, 126-147.

Logan, G. D., \& Bundesen, C. (2004). Very clever homunculus: Compound stimulus strategies for the explicit task-cuing procedure. Psychonomic Bulletin \& Review, 11, 832-840.

Logan, G. D., \& SchneIder, D. W. (2006). Interpreting instructional cues in task switching procedures: The role of mediator retrieval Journal of Experimental Psychology: Learning, Memory, \& Cognition, 32, 347-363.

Los, S. A. (1996). On the origin of mixing costs: Exploring information processing in pure and mixed blocks of trials. Acta Psychologica, 94, $145-188$.

MAYr, U. (2003). Towards principles of executive control: How mental sets are selected. In R. H. Kluwe, G. Luer, \& F. Rosler (Eds.), Principles of learning and memory (pp. 223-240). Basel: Birkhäuser.

MeIRAn, N. (1996). Reconfiguration of processing mode prior to task performance. Journal of Experimental Psychology: Learning, Memory, \& Cognition, 22, 1423-1442.

Meiran, N., Chorev, Z., \& SaPIR, A. (2000). Component processes in task switching. Cognitive Psychology, 41, 211-253.

Miyake, A., Emerson, M. J., Padilla, F., \& Ahn, J.-C. (2004). Inner speech as a retrieval aid for task goals: The effects of cue type and articulatory suppression in the random task cuing paradigm. Acta Psychologica, 115, 123-142.

Monsell, S. (2003). Task switching. Trends in Cognitive Sciences, 7, 134-140.
Rogers, R. D., \& Monsell, S. (1995). Costs of a predictable switch between simple cognitive tasks. Journal of Experimental Psychology: General, 124, 207-231.

Rubin, O., \& Meiran, N. (2005). On the origins of the task mixing cost in the cuing task-switching paradigm. Journal of Experimental Psychology: Learning, Memory, \& Cognition, 31, 1477-1491.

Rushworth, M. F. S., Hadland, K. A., Paus, T., \& Sipila, P. K. (2002). Role of the human medial frontal cortex in task switching: A combined fMRI and TMS study. Journal of Neurophysiology, 87, 2577-2592.

SAEKI, E., \& SAITO, S. (2004a). Effect of articulatory suppression on task-switching performance: Implications for models of working memory. Memory, 12, 257-271.

SAEKI, E., \& SAITO, S. (2004b). The role of the phonological loop in task switching performance: The effect of articulatory suppression in the alternating runs paradigm. Psychologia, 47, 35-43.

Saeki, E., Saito, S., \& Kawaguchi, J. (2006). Effect of responsestimulus interval manipulation and articulatory suppression on task switching. Memory, 14, 965-976.

SchneIder, D. W., \& Logan, G. D. (2007). Task switching versus cue switching: Using transition cuing to disentangle sequential effects in task-switching performance. Journal of Experimental Psychology: Learning, Memory, \& Cognition, 33, 370-378.

(Manuscript received December 21, 2008; revision accepted for publication May 11, 2009.) 